\title{
PLANAR VECTOR FIELDS WITH A GIVEN SET OF ORBITS
}

\author{
JAUME LLIBRE ${ }^{1}$, RAFAEL RAMÍREZ ${ }^{2}$ AND NATALIA SADOVSKAIA ${ }^{3}$
}

\begin{abstract}
We determine all the $\mathcal{C}^{1}$ planar vector fields with a given set of orbits of the form $y-y(x)=0$ satisfying convenient assumptions. The case when these orbits are branches of an algebraic curve is also study. We show that if a quadratic vector field admits a unique irreducible invariant algebraic curve $g(x, y)=\sum_{j=0}^{S} a_{j}(x) y^{S-j}=0$ with $S$ branches with respect
\end{abstract} to the variable $y$, then the degree of the polynomial $g$ is at most $4 S$.

\section{IntRoduCtion AND STATEMENT OF THE MAIN RESUltS}

By definition an autonomous complex planar differential system is a system of the form

$$
\dot{\mathbf{x}}=\mathcal{X}(\mathbf{x}), \quad \mathbf{x}=(x, y) \in \mathrm{D} \subseteq \mathbb{C}^{2},
$$

where the dependent variables $\mathbf{x}=(x, y)$ are complex, and the independent variable (time $t$ ) is real. We assume that the vector field $\mathcal{X}=(P, Q)$ associated to the differential system $(1)$ is $\mathcal{C}^{1}$ in an open subset $\mathrm{D}$ of $\mathbb{C}^{2}$.

Let $g=g(\mathbf{x})$ be a $\mathcal{C}^{1}$ function. The curve $\tilde{g}=0$ is an invariant curve of the vector field $\mathcal{X}$ if

$$
\left.\mathcal{X} g\right|_{g=0}=0,
$$

i.e. the curve $g=0$ is formed by orbits of $\mathcal{X}$.

The vector field $\mathcal{X}$ is called a polynomial vector field of degree $n$ if $P$ and $Q$ are polynomials such that the maximum of the degrees of $P$ and $Q$ is $n$. Let $g$ be a complex polynomial in the variables $x$ and $y$ irreducible in the ring of polynomials $\mathbb{C}[x, y]$. Suppose that $g$ satisfies (2), then we say that $g=0$ is an invariant algebraic curve of $\mathcal{X}$. By the Hilbert's Nullstellensatz Theorem [6] if the polynomial $\mathcal{X}(g)$ vanishes when $g=0$, there exist a non-negative integer $m$ and a polynomial $M=M(x, y)$ such that $(\mathcal{X}(g))^{m}=M g$. Since $g$ is irreducible, then there exist a polynomial $K=K(x, y)$ such that $\mathcal{X}(g)=K g$, the polynomial $K$ is called the cofactor of $g=0$, and clearly the degree of $K$ is at most $n-1$.

We shall present briefly the contents of the paper.

1991 Mathematics Subject Classification. Primary 34C05, 34A34, 34C14.

Key words and phrases. invariant curve, singular algebraic curve, branches, quadratic vector fields, orthogonal polynomial, orbits. 
In section 2 we prove our first main result (see Theorem 1 below) and determine a planar vector field from a given set of invariant orbits of the form $y-y(x)=0$ where $y(x)$ is an arbitrary $\mathcal{C}^{1}$ function, and consequently the vector fields having such orbits are in general $\mathcal{C}^{1}$ vector fields.

In section 3 we apply Theorem 1 to the set of orbits which are branches of an algebraic curve $g(x, y)=0$ (see Proposition 10).

In section 4 we prove our second main result for the vector fields of degree two (see Theorem 3). More precisely for such vector fields having a unique irreducible invariant algebraic curve $g=0$ we bound the degree of $g$ by four times the number of its branches with respect to the variable $x$ or $y$. The result is written with respect to the variable $y$.

In section 5 we determine the $\mathcal{C}^{1}$ planar vector field with only one invariant curve of the form $a_{0}(x) y+a_{1}(x)=0$ (see Proposition 14).

In section 6 as application of our previous results given in section 5 we determine the polynomial planar vector fields of degree 2,3 or 4 , with only one invariant algebraic curve of the type $g=f^{\prime}(x) y+f(x)=0$ or $g=$ $f(x) y+f^{\prime}(x)=0$ where $f$ is an orthogonal polynomial (see Proposition 17).

Finally in section 7 we analyze 16th Hilbert problem for limit cycles on a singular invariant algebraic curve, i.e. an invariant algebraic curve $g=0$ having in the complex projective plane points such that the curve and its first derivatives are zero.

Our first main result is the following.

Theorem 1. Let

$$
g_{j}(x, y)=y-y_{j}(x)=0, \quad j=1,2, \ldots, S \geq 2,
$$

be a given set of orbits not formed by singular points of a complex planar differential system $(\mathcal{S})$, where $y_{j}=y_{j}(x)$ is a $\mathcal{C}^{1}$ function for $j=1, \ldots, S$ such that

(4)

$$
\triangle_{0}=\Delta_{0}(x)=\left|\begin{array}{lllll}
1 & 1 & \ldots & \ldots & 1 \\
y_{1} & y_{2} & \ldots & \ldots & y_{S} \\
\vdots & \vdots & \vdots & \vdots & \vdots \\
y_{1}^{S-1} & y_{2}^{S-1} & \ldots & \ldots & y_{S}^{S-1}
\end{array}\right|=\prod_{1 \leq m<j \leq S}\left(y_{m}-y_{j}\right) \neq 0
$$

and there are at least two functions $g_{1}$ and $g_{2}$ for which

$$
\left\{g_{1}, g_{2}\right\}=y_{2}^{\prime}-y_{1}^{\prime} \neq 0 .
$$


Then the planar differential system $(\mathcal{S})$ can be written as

$$
\begin{aligned}
& \dot{x}=\sum_{j=1}^{S} \lambda_{j} \prod_{\substack{m=1 \\
m \neq j}}^{S}\left(y-y_{m}\right)+\lambda_{S+2} g=P(x, y), \\
& \dot{y}=\sum_{j=1}^{S} \lambda_{j} y_{j}^{\prime} \prod_{\substack{m=1 \\
m \neq j}}^{S}\left(y-y_{m}\right)-\lambda_{S+1} g=Q(x, y),
\end{aligned}
$$

where $g=\prod_{m=1}^{S}\left(y-y_{m}\right)$ and $\lambda_{j}=\lambda_{j}(x, y)$ for $j=1,2, \ldots, S$ are arbitrary $\mathcal{C}^{1}$ functions.

It is well known (see for instance [5]) that if $g$ is a polynomial, then the polynomial differential system

$$
\dot{x}=\lambda \frac{\partial g}{\partial y}+\mu_{1} g, \quad \dot{y}=-\lambda \frac{\partial g}{\partial x}+\mu_{2} g,
$$

where $\lambda, \mu_{1}$ and $\mu_{2}$ are arbitrary polynomials on $x$ and $y$, has $g=0$ as an invariant algebraic curve.

Proposition 2. The polynomial system (7) can be written in the form (6).

We denote the degree of a polynomial $g$ by $\operatorname{deg} g$. For the definition of branches of an algebraic curve see the beginning of section 3. Our second main result is the following.

Theorem 3. Let $X$ be the quadratic vector field associated to the quadratic system

$$
\dot{x}=p_{0} y^{2}+p_{1} y+p_{2}, \quad \dot{y}=q_{0} y^{2}+q_{1} y+q_{2},
$$

where $p_{j}=p_{j}(x)=\sum_{n=0}^{j} p_{j n} x^{n}, q_{j}=q_{j}(x)=\sum_{n=0}^{j} q_{j n} x^{n}$, for $j=1,2$, and for which

$$
g=\sum_{j=0}^{S} a_{j}(x) y^{S-j}=0
$$

is the unique irreducible invariant algebraic curve. If the curve $g=0$ has $S>1$ branches with respect to the variable $y\left(\right.$ so $\left.a_{0} \neq 0\right)$. Then $\operatorname{deg} g \leq 4 S$.

We introduce the following two conjectures which are commented in Remark 13 and in the paragraphs following this remark.

Conjecture 4. If a quadratic polynomial differential system (8) admits a unique invariant irreducible algebraic curve $g=0$ given in (9), then $\operatorname{deg} g \leq$ $3 S$. 
Conjecture 5. If a quadratic polynomial differential system (8) with a unique invariant irreducible algebraic curve $g=0$ given in (9) does not admit a rational first integral, then $\operatorname{deg} g \leq 12$.

\section{2. $C^{1}$ VECTOR FIELDS With AT LEAST TWO INVARIANT CURVES OF THE FORM $y=y(x)$}

Proof of Theorem 1. Let $\mathcal{X}=(P, Q)$ be the vector field associated to differential system (6). Now we shall prove that the given orbits (3) are invariant curves of (6). Indeed the vector field $\mathcal{X}$ admits the equivalent representation (see also [13]).

$$
\mathcal{X}(*)=\sum_{j=1}^{S+2} \lambda_{j} \prod_{\substack{m=1 \\ m \neq j}}^{S} g_{m}\left\{*, g_{j}\right\}
$$

where $g_{S+1}=x, g_{S+2}=y$, i.e. $\dot{x}=\mathcal{X}(x), \dot{y}=\mathcal{X}(y)$. Hence

$$
\mathcal{X}\left(g_{l}\right)=g_{l}\left(\sum_{\substack{m=1 \\ m \neq l}}^{S+2} \lambda_{j} \prod_{\substack{m=1 \\ m \neq j}}^{S} g_{m}\left\{g_{l}, g_{j}\right\}\right) \equiv \Phi_{l}, \quad l=1, \ldots, S
$$

i.e., $g_{l}=y-y_{l}=0$ is an invariant curve of $\mathcal{X}$.

We shall show that $\mathcal{X}$ is the most general planar vector field having the invariant curves $g_{l}=0$ for $l=1,2, \ldots, S$ satisfying (4) and (5). Let $\mathcal{Y}=$ $\left(Y_{1}(x, y), Y_{2}(x, y)\right)$ be a planar vector field with the given invariant curves satisfying (4) and (5). We look for functions $\lambda_{j}$ for $j=1,2, \ldots, S+2$ satisfying

$$
\begin{aligned}
& \sum_{j=1}^{S} \lambda_{j} \prod_{\substack{m=1 \\
m \neq j}}^{S}\left(y-y_{m}\right)+\lambda_{S+2} g=Y_{1}, \\
& \sum_{j=1}^{S} \lambda_{j} y_{j}^{\prime} \prod_{\substack{m=1 \\
m \neq j}}^{S}\left(y-y_{m}\right)-\lambda_{S+1} g=Y_{2} .
\end{aligned}
$$

Therefore taking $y=y_{j}$ in these expressions we obtain

$$
\begin{aligned}
& \lambda_{j}\left(x, y_{j}\right) \prod_{\substack{m=1 \\
m \neq j}}^{S}\left(y_{j}(x)-y_{m}(x)\right)=Y_{1}\left(x, y_{j}\right), \\
& \lambda_{j}\left(x, y_{j}\right) y_{j}^{\prime}(x) \prod_{\substack{m=1 \\
m \neq j}}^{S}\left(y_{j}(x)-y_{m}(x)\right)=Y_{2}\left(x, y_{j}\right),
\end{aligned}
$$

or equivalently

$$
\begin{aligned}
& \lambda_{j}\left(x, y_{j}\right)=(-1)^{S-j-1} \frac{\Delta_{j}}{\Delta_{0}} Y_{1}\left(x, y_{j}\right), \\
& \lambda_{j}\left(x, y_{j}\right) y_{j}^{\prime}=(-1)^{S-j-1} \frac{\Delta_{j}}{\Delta_{0}} Y_{2}\left(x, y_{j}\right) .
\end{aligned}
$$


Here we have used assumption (4), where

$$
\triangle_{j}=\Delta_{j}(x)=\left|\begin{array}{llllll}
1 & \ldots & 1 & 1 & \vdots & 1 \\
y_{1} & \ldots & y_{j-1} & y_{j+1} & \vdots & y_{S} \\
\vdots & \ldots & \vdots & \vdots & & \\
y_{1}^{S-2} & \ldots & y_{j-1}^{S-2} & y_{j+1}^{S-2} & \vdots & y_{S}^{S-2}
\end{array}\right|
$$

for $j=1,2, \ldots S$.

Solving system (10) with respect to $\lambda_{1}$ and $\lambda_{2}$ we get that

$$
\begin{gathered}
\lambda_{1}=\lambda_{1}(x, y)=\frac{\left(Y_{1}-\Psi_{1}\right) y_{2}^{\prime}-\left(Y_{2}-\Psi_{2}\right)}{\left(y_{2}^{\prime}-y_{1}^{\prime}\right) \prod_{m=2}^{S}\left(y-y_{m}\right)}, \\
\lambda_{2}=\lambda_{2}(x, y)=-\frac{\left(Y_{1}-\Psi_{1}\right) y_{1}^{\prime}-\left(Y_{2}-\Psi_{2}\right)}{\left(y_{2}^{\prime}-y_{1}^{\prime}\right) \prod_{\substack{m=1 \\
m \neq 2}}^{S}\left(y-y_{m}\right)},
\end{gathered}
$$

where

$$
\Psi_{1}=\sum_{j=3}^{S} \lambda_{j} \prod_{\substack{m=1 \\ m \neq j}}^{S}\left(y-y_{m}\right)+\lambda_{S+2} g, \quad \Psi_{2}=\sum_{j=3}^{S} \lambda_{j} y_{j}^{\prime} \prod_{\substack{m=1 \\ m \neq j}}^{S}\left(y-y_{m}\right)-\lambda_{S+1} g
$$

and $y_{2}^{\prime}-y_{1}^{\prime} \neq 0$ by assumption (5). The expressions

$$
\prod_{m=2}^{S}\left(y-y_{m}\right) \quad \text { and } \quad \prod_{\substack{m=1 \\ m \neq 2}}^{S}\left(y-y_{m}\right)
$$

which appear in the denominator of $\lambda_{1}$ and $\lambda_{2}$ in (12), do not provide problems in the definition of $\lambda_{1}$ and $\lambda_{2}$, because when we evaluate $\lambda_{1}(x, y)$ and $\lambda_{2}(x, y)$ in $y=y_{j}(x)$ for any $j=1,2, \ldots, S$ using the expression (11), then the factor $y_{j}-y_{j}=0$ also appears in the numerator. So $\lambda_{1}$ and $\lambda_{2}$ are well defined.

Substituting $\lambda_{1}$ and $\lambda_{2}$ in (6) we obtain the vector field $\mathcal{Y}$. Hence Theorem 1 is proved.

Remark 6. We recall that the determinant $\Delta_{0}$ is usually called a Vandermonde determinant.

Remark 7. The natural $S \geq 2$ in Theorem 1 is arbitrary.

Remark 8. Let $\left(x_{0}, y_{0}\right)$ be an intersection point of two curves $g_{j}=y-y_{j}=0$ and $g_{k}=y-y_{k}=0$ with $j \neq k$, then this point is a singular point of system (6), and on it $\Delta_{0}=0$. Moreover from (4) these points are the unique where $\Delta_{0}$ vanishes. 
Corollary 9 (see $[14,15])$. Under the assumptions of Theorem 1 system (6) can be written as

$$
\begin{gathered}
\dot{x}=\left|\begin{array}{cccc}
1 & 1 & \ldots & 1 \\
y & y_{1} & \ldots & y_{S} \\
\vdots & \vdots & \vdots & \vdots \\
y^{S-1} & y_{1}^{S-1} & \ldots & y_{S}^{S-1} \\
\tilde{\lambda}_{S+2} y^{S} & \tilde{\lambda}_{S+2} y_{1}^{S}+\tilde{\lambda}_{1} & \ldots & \tilde{\lambda}_{S+2} y_{S}^{S}+\tilde{\lambda}_{S}
\end{array}\right|, \\
\dot{y}=\left|\begin{array}{cccc}
1 & 1 & \ldots & 1 \\
y & y_{1} & \ldots & y_{S} \\
\vdots & \vdots & \vdots & \vdots \\
y^{S-1} & y_{1}^{S-1} & \ldots & y_{S}^{S-1} \\
\tilde{\lambda}_{S+1} y^{S} & \tilde{\lambda}_{S+1} y_{1}^{S}+\tilde{\lambda}_{1} y_{1}^{\prime}(x) & \ldots & \tilde{\lambda}_{S+1} y_{S}^{S}+\tilde{\lambda}_{S} y_{S}^{\prime}(x)
\end{array}\right|,
\end{gathered}
$$

where

$$
\begin{gathered}
\tilde{\lambda}_{S+k} \prod_{1 \leq i \leq j \leq S}\left(y_{j}-y_{i}\right)=\lambda_{S+k}, \\
\tilde{\lambda}_{m}(-1)^{S-m+1} \prod_{\substack{1 \leq i \leq j \leq S \\
i, j \neq m}}\left(y_{j}-y_{i}\right)=\lambda_{m},
\end{gathered}
$$

for $k=1,2$ and $m=1,2, \ldots, S$.

Proof. Developing by the last row the determinants of the statement of the corollary we get system (6).

\section{Polynomial Vector fields With invariant algebraic CuRVes WITH AT LEAST TWO BRANCHES}

In the rest of this paper we shall work with complex polynomial vector fields. First we shall study the planar polynomial vector field $\mathcal{X}$ of degree $n$ having the invariant algebraic curve

$$
g=\sum_{j=0}^{S} a_{j}(x) y^{S-j}
$$

where $a_{j}=a_{j}(x)$ for $j=0, \ldots, S$ are polynomials. If $a_{0}(x) \neq 0$, then it is well known

$$
g=a_{0}(x) \prod_{j=1}^{S}\left(y-y_{j}(x)\right)=0
$$

where $y_{j}=y_{j}(x)$, for $j=1,2, \ldots, S$ are algebraic functions. Moreover

$$
a_{1}=-a_{0} \sum_{j=1}^{S} y_{j}, \quad a_{2}=a_{0} \sum_{1 \leq j<m \leq S} y_{j} y_{m}, \quad \cdots \quad, a_{S}=(-1)^{S} a_{0} \prod_{j=1}^{S} y_{j} .
$$

The functions $g_{j}=y-y_{j}$ for $j=1, \ldots, S$ are called the branches of the algebraic curve $g=0$ with respect to the variable $y$. 
Proposition 10. Let (13) be the product of all the invariant algebraic curves of a polynomial vector field $\mathcal{X}$ of degree $n$. Then the branches $g_{j}=y-y_{j}(x)=0$ for $j=1,2, \ldots, S$ of $g=0$ are invariant curves of the vector field $\mathcal{X}$.

Proof. Using the branches $g_{j}=y-y_{j}=0$ of $g=0$ given by (14) we can write the vector field $\mathcal{X}$ in the form given by (6). ¿From Theorem 1 the proposition follows.

Remark 11. There are polynomial vector fields with an invariant algebraic curve having an arbitrary number of branches, this follows from Remark 3 and from the fact that the branches of an invariant algebraic curve are invariant curves of the vector field $\mathcal{X}$ (see Proposition 10).

Proof of Proposition 2. Choosing in (6) the arbitrary functions $\lambda_{j}$ as follows

$$
\lambda_{1}=\lambda_{2}=\ldots=\lambda_{S}=\lambda, \quad \lambda_{S+1}=-\mu_{2}, \quad \lambda_{S+2}=\mu_{1},
$$

we obtain that system (6) becomes

$$
\begin{aligned}
& \dot{x}=\lambda \sum_{j=1}^{S} \prod_{\substack{m=1 \\
m \neq j}}^{S}\left(y-y_{m}(x)\right)+\lambda_{S+2} g=\lambda \frac{\partial g}{\partial y}+\mu_{1} g, \\
& \dot{y}=-\lambda \sum_{j=1}^{S} y_{j}^{\prime}(x) \prod_{\substack{m=1 \\
m \neq j}}^{S}\left(y-y_{m}(x)\right)-\lambda_{S+1} g=-\lambda \frac{\partial g}{\partial x}+\mu_{2} g .
\end{aligned}
$$

We note that the curve $g=0$ of system (7) is not necessarily irreducible.

Remark 12. It is well known that if the invariant algebraic curve of a polynomial differential system of degree $n$ is nonsingular in $\mathbb{C} P^{2}$, then $\operatorname{deg} g \leq n+1$ (see for instance Corollary 4 of [4] and Theorem 2 of [16]). As a consequence if $\operatorname{deg} g>n+1$, then this curve is singular in $\mathbb{C} P^{2}$

\section{QUADRATIC SYSTEM With A UNIQUE IRREDUCIBLE INVARIANT ALGEBRAIC CURVE}

In this section we study the quadratic systems, i.e. polynomial differential systems of degree 2 .

Proof of Theorem 3. From the relation $X g=(\alpha y+\beta x+\gamma) g$, taking the coefficients of the powers of $y$ we obtain the following differential system

$$
p_{0} \frac{d a_{0}}{d x}=0, \quad A \frac{d \mathbf{a}}{d x}=B \mathbf{a}, \quad p_{2} \frac{d a_{S}}{d x}=(\beta x+\gamma) a_{S}-q_{2} a_{S-1},
$$


where $\mathbf{a}=\left(a_{0}, a_{1}, \ldots, a_{S}\right)^{T}$, and $a_{i}=a_{i}(x)$ for $i=0,1, \ldots, S$, and $A$ and $B$ are the following $(S+1) \times(S+1)$ matrices

$$
\begin{aligned}
A & =\left(\begin{array}{cccccccccc}
p_{1} & p_{0} & 0 & 0 & 0 & \ldots & 0 & 0 & 0 & 0 \\
p_{2} & p_{1} & p_{0} & 0 & 0 & \ldots & 0 & 0 & 0 & 0 \\
0 & p_{2} & p_{1} & p_{0} & 0 & \ldots & 0 & 0 & 0 & 0 \\
0 & 0 & p_{2} & p_{1} & p_{0} & \ldots & 0 & 0 & 0 & 0 \\
\vdots & \vdots & \vdots & \vdots & \vdots & \vdots & \vdots & \vdots & \vdots & \vdots \\
0 & 0 & 0 & 0 & 0 & \ldots & 0 & p_{2} & p_{1} & p_{0} \\
0 & 0 & 0 & 0 & 0 & \ldots & 0 & 0 & p_{2} & p_{1}
\end{array}\right) \\
B & =\left(\begin{array}{cccccccccc}
\tilde{a}_{0} & 0 & 0 & 0 & \ldots & 0 & 0 & 0 & 0 \\
b_{0} & \tilde{a}_{1} & 0 & 0 & \ldots & 0 & 0 & 0 & 0 \\
c_{0} & b_{1} & \tilde{a}_{2} & 0 & \ldots & 0 & 0 & 0 & 0 \\
0 & c_{1} & b_{2} & \tilde{a}_{3} & \ldots & 0 & 0 & 0 & 0 \\
\vdots & \vdots & \vdots & \vdots & \vdots & \vdots & \vdots & \vdots & \vdots \\
0 & 0 & 0 & 0 & \ldots & c_{S-3} & b_{S-2} & \tilde{a}_{S-1} & 0 \\
0 & 0 & 0 & 0 & \ldots & 0 & c_{S-2} & b_{S-1} & \tilde{a}_{S}
\end{array}\right),
\end{aligned}
$$

here $\tilde{a}_{j}=\alpha+(j-S) q_{0}, b_{j}=\beta x+\gamma+(j-S) q_{1}, c_{j}=(j-S) q_{2}$ for $j=0,1, \ldots, S$. It is known (see for instance [7]) that after a linear change of variables and a rescaling of the time any quadratic system (8) can be written as

$$
\dot{x}=P(x, y), \quad \dot{y}=q_{0} y^{2}+q_{1} y+q_{2},
$$

where $P(x, y)$ is one of the following ten polynomials

$$
1+x y, \quad y+x^{2}, \quad y, \quad 1, \quad x y, \quad-1+x^{2}, \quad 1+x^{2}, \quad x^{2}, \quad x, \quad 0 .
$$

Since the last six possibilities for $P(x, y)$ force that the quadratic system has an invariant straight line (real or complex) and by assumption our quadratic system has no invariant straight lines, the polynomial $P(x, y)$ only can be $1+x y, y+x^{2}, y, 1$.

Case 1: Assume that $P$ is either $y+x^{2}$, or $y$. We consider the quadratic system

$$
\dot{x}=y+p_{2}(x), \quad \dot{y}=q_{0} y^{2}+q_{1} y+q_{2},
$$

with $p_{2}(x)=x^{2}$ or $p_{2}(x)=0$. After the recursive integration of system (15), since the $a_{j}$ 's are polynomials, we deduce that

$$
\begin{aligned}
& \alpha=S q_{0}, \\
& a_{0}=a_{00}, \\
& a_{1}=a_{12} x^{2}+a_{11} x+a_{10}, \\
& a_{2}=a_{24} x^{4}+a_{23} x^{3}+a_{22} x^{2}+a_{21} x+a_{20}, \\
& \vdots \\
& a_{S-1}=a_{S-1,2(S-1)} x^{2(S-1)}+\ldots, \\
& a_{S}=a_{S, 2 S} x^{2 S}+\ldots,
\end{aligned}
$$

where all the $a_{i j}$ are constants. Therefore $\operatorname{deg} g \leq 2 S$. 
Case 2: Assume that $P=1$. Now we deal with the quadratic system

$$
\dot{x}=1, \quad \dot{y}=q_{0} y^{2}+q_{1} y+q_{2} .
$$

We note that the previous differential system can be written as a Ricatti differential equation. Since this system has no singular points, the algebraic invariant curve $g=0$ must be non-singular in the affine plane. If the curve is nonsingular in $\mathbb{C} P^{2}$ then the degree of $g$ is at most three (see remark 12). So if the algebraic curve $g=0$ of (16) has degree larger than three, it is nonsingular in the affine plane and singular at infinity, i.e. in $\mathbb{C} P^{2}$. We shall determine the curve $g=0$ solution of (15) with degree $>3$.

Assume that $q_{11} \neq 0$. After the change of variables $\left(q_{11} x, y\right) \longrightarrow(y, x)$ and introducing the notations

$$
\frac{q_{22}}{q_{11}^{2}}=p_{0}, \quad \frac{q_{21}}{q_{11}}=p_{11}, \quad p_{2}(x)=q_{0} x^{2}+q_{10} x+q_{20},
$$

we obtain the system

$$
\dot{x}=p_{0} y^{2}+x y+p_{11} y+p_{2}(x), \quad \dot{y}=q_{11},
$$

We consider the differential system (15) associated to system (17). If $p_{0} \neq 0$ without loss of generality we can take $p_{0}=1$. Then system (15) takes the form

$$
\begin{aligned}
a_{0}^{\prime}= & 0, \\
a_{1}^{\prime}= & m a_{0}, \\
a_{2}^{\prime}= & \left(m^{2}+m\left(q_{0}-q_{11}\right)+\beta-s q_{11}\right) x+ \\
& \left(m\left(C_{1}-p_{10} C_{0}\right)+q_{0} C_{1}+\left(\gamma-S q_{11}\right) C_{0},\right.
\end{aligned}
$$

Hence we obtain that $\operatorname{deg} a_{j} \leq j$, for $j=0,1, \ldots S$, and consequently $\operatorname{deg} g \leq S$.

We study the case $p_{0}=0$. Therefore the differential system (15) is

$$
\begin{aligned}
x a_{0}^{\prime} & =\alpha a_{0}, \\
x a_{j+1}^{\prime} & =\alpha a_{j+1}+(\beta x+\gamma) a_{j}-\left(p_{22} x^{2}+p_{21} x+p_{20}\right) a_{j}^{\prime}-q_{11}(S+1-j) a_{j-1},
\end{aligned}
$$

for $j=0, \ldots, S$, where $a_{-1}=0$. Solving the first differential equation we get $a_{0}=C_{0} x^{\alpha}$, hence $\alpha$ must be a non-negative integer, and without loss of generality we can take $C_{0}=1$. Now substituting it into the differential equation of $a_{1}^{\prime}$ we obtain

$$
\begin{aligned}
x a_{1}^{\prime} & =\alpha a_{1}+(\beta x+\gamma) x^{\alpha}-\alpha\left(p_{22} x^{2}+p_{21} x+p_{20}\right) x^{\alpha-1} \\
& =\alpha a_{1}+\left(\beta-\alpha p_{22}\right) x^{\alpha+1}+\left(\gamma-\alpha p_{21}\right) x^{\alpha}-\alpha p_{20} x^{\alpha-1} .
\end{aligned}
$$

Solving this linear differential equation we have

$$
a_{1}=\left(\beta-\alpha p_{22}\right) x^{\alpha+1}+C_{1} x^{\alpha}+\alpha p_{20} x^{\alpha-1}+\left(\gamma-\alpha p_{21}\right) x^{\alpha} \log x .
$$

Since $a_{1}$ must be a polynomial we get that

$$
\gamma=\alpha p_{21} \text {. }
$$


Solving the differential equation of $a_{2}^{\prime}$ we obtain

$$
\begin{aligned}
a_{2}(x)= & \alpha p_{20}^{2}(\alpha-1) x^{\alpha-2}+\alpha p_{20}\left(p_{21}-C_{1}\right) x^{\alpha-1}+C_{2} x^{\alpha}- \\
& \left(C_{1}-p_{21}\right)\left(\alpha p_{22}-\beta\right) x^{\alpha+1}+\frac{1}{2}\left(\alpha p_{22}-\beta\right)\left((\alpha+1) p_{22}-\beta\right) x^{\alpha+2}- \\
& \left(S q_{11}-\left(2 \alpha p_{22}-\beta\right) p_{20} x^{\alpha} \log x\right.
\end{aligned}
$$

Again since $a_{2}$ must be a polynomial we get that

$$
S=\frac{p_{20}\left(2 \alpha p_{22}-\beta\right)}{q_{11}} .
$$

Doing similar arguments and considering that we can write

$$
\begin{aligned}
& (\beta x+\gamma) a_{j}-\left(p_{22} x^{2}+p_{21} x+p_{20}\right) a_{j}^{\prime}-q_{11}(S+1-j) a_{j-1}= \\
& \left(-q_{11}(S-j) C_{j-1}+\ldots\right) x^{\alpha}+\ldots,
\end{aligned}
$$

for $j \geq 3$, we can obtain solving the linear differential equation for $a_{j}^{\prime}$ that all $a_{j}$ for $j \geq 3$ are polynomials choosing the arbitrary constant $C_{j-1}$ conveniently.

After the recursive integrations we finally deduce that

$$
\begin{aligned}
a_{j} & =\prod_{m=1}^{j}\left(\beta-(-1+m+\alpha) p_{22}\right) \frac{x^{\alpha+j}}{j !}+x^{\alpha-j} P_{2 j-1}(x) \\
& =x^{\alpha-j}\left(\prod_{m=1}^{j}\left(\beta-(-1+m+\alpha) p_{22}\right) \frac{x^{2 j}}{j !}+P_{2 j-1}(x)\right),
\end{aligned}
$$

where $P_{m}(x)$ is a polynomial of degree $m$ in $x$ and by definition $P_{-1}(x)=0$. The invariant algebraic curve in this case admits the representation

$$
g=x^{\alpha-S} \sum_{j=0}^{S}(x y)^{S-j}\left(\prod_{m=1}^{j}\left(\beta-(-1+m+\alpha) p_{22}\right) \frac{x^{2 j}}{j !}+P_{2 j-1}(x)\right) .
$$

Hence $\operatorname{deg} g \leq \alpha+S$.

If $\alpha-S \geq 0$ then by considering that the curve is irreducible, we have $\alpha=S$, and as a consequence $\operatorname{deg} g \leq 2 S$. If $\alpha-S<0$ then $\operatorname{deg} g \leq \alpha+S<2 S$. In short in case 2 and when $q_{11} \neq 0$ we have that $\operatorname{deg} g \leq 2 S$. Substituting $a_{S}$ and $a_{S-1}$ in the last equation of (15) and taking the biggest coefficient in $x$ (i.e. the coefficient of $x^{\alpha+S+1}$ ) we deduce that

$$
\prod_{m=1}^{S+1}\left(\beta-(1-m+\alpha) p_{22}\right)=0 .
$$

It is interesting the particular case 2 with $q_{11} \neq 0$ when $S>\alpha=1$ and $\beta=p_{22} \neq 0, p_{20}=\gamma=0$. The solutions of (15) are polynomial of degree one of the form $a_{j}=c_{j} x+r_{j}$, for $j=0,1, \ldots, S$ where $c_{j}$ and $r_{j}$ are convenient constants satisfying the equations

$r_{j+1}=p_{20} c_{j}+(S+1-j) q_{11} r_{j-1}, \quad p_{22} r_{j}=q_{11}(S+1-j) c_{j-1}, \quad r_{-1}=c_{-1}=0$, 
for $j=0,1, \ldots, S$. Hence we obtain that

$$
\begin{aligned}
& p_{22} p_{20}=S q_{11}, \\
& r_{2 j}=0, \quad r_{1}=p_{20}, \quad r_{2 j+1}=\frac{p_{20}(S-2 j)}{S} c_{2 j}, \\
& c_{2 j+1}=0, \quad c_{0}=1, \quad c_{2 j}=\frac{\left(-q_{11}\right)^{j} S !}{2^{j} j !(S-2 j) !} .
\end{aligned}
$$

Consequently the curve $g=0$ takes the form

$$
g=\sum_{k=0}^{S} a_{k}(x) y^{S-k}=x \sum_{k=0}^{S} c_{k} y^{S-k}+\sum_{k=0}^{S} r_{k} y^{S-k}
$$

or equivalently

$$
g=x \sum_{k=0}^{[S / 2]} c_{2 k} y^{S-2 k}+\sum_{k=0}^{[(S-1) / 2]} r_{2 k+1} y^{S-2 k-1},
$$

where $[x]$ is the integer part function of the real number $x$.

If we denote

$$
H_{S}(y)=\sum_{k=1}^{[S / 2]} c_{2 k}\left(q_{11} y\right)^{S-2 k}=\sum_{k=0}^{[S / 2]} \frac{\left(-q_{11}\right)^{k} S !}{2^{k} k !(S-2 k) !}\left(q_{11} y\right)^{S-2 k},
$$

then we obtain the following representation for $g$

$$
g(x, y)=x H_{S}(y)+\frac{p_{20}}{S} H_{S}^{\prime}(y)
$$

It is easy to check that if if $q_{11}=2$, then $H_{S}$ coincide with the physicists' Hermite polynomial. Clearly $\operatorname{deg} g=S+1$.

Now we assume that in (16) $q_{11}=0$ and $q_{21} \neq 0$, then doing the change of variables $\left(q_{21} x, y\right) \mapsto(y, x)$ we obtain

$$
\dot{x}=p_{0} y^{2}+y+p_{2}(x), \quad \dot{y}=q_{21},
$$

where $p_{0}=q_{22} / q_{21}^{2}, p_{2}(x)=q_{0} x^{2}+q_{10} x+q_{20}$. If $p_{0}=q_{22} \neq 0$, then system (15) admits the polynomial solutions

$$
a_{0}=a_{00}, \quad a_{1}=a_{11} x+a_{10}, \quad \ldots a_{S}=a_{S S} x^{S}+\ldots+a_{S 0},
$$

so $\operatorname{deg} g \leq S$. If $p_{0}=q_{22}=0$, then the integration of equation (15) is analogous to case 1 . Hence $\operatorname{deg} g \leq 2 S$.

Case 3: Assume that $P=x y+1$. Finally we must study the quadratic systems

$$
\dot{x}=x y+1, \quad \dot{y}=q_{0} y^{2}+\left(q_{11} x+q_{10}\right) y+q_{22} x^{2}+q_{21} x+q_{20} .
$$

If $\alpha-S q_{0}=m$ we shall show that the functions $a_{j}(x)$ of (15) are polynomials of degree $\operatorname{deg} a_{j} \leq q_{0} j+m$ if $q_{0} \neq 0$, and of $\operatorname{deg} a_{j} \leq j+m$ if $q_{0}=0$, for $j=0,1, \ldots S$.

We denote $q_{0}=k$. Since $p_{0}=0, p_{1}=x, p_{2}=1$, from the first nonzero differential equation of system (15) we obtain that $a_{0}=x^{m} C_{0}$ with $C_{0} \in$ 
$\mathbb{C} \backslash\{0\}$. Since $a_{0}(x)$ must be a polynomial, $m$ must be a non-negative integer, i.e. $\alpha-S q_{0}=m \geq 0$ as a consequence $S q_{0} \leq \alpha$.

Solving the second nonzero differential equation of system (15) we obtain

$$
\begin{aligned}
a_{1}(x) & =C_{0}\left(\frac{4 q_{11}-\beta}{k-1} x^{m+1}+\frac{4 q_{10}-\gamma}{k} x^{m}+\frac{m}{1+k} x^{m-1}\right)+C_{1} x^{m+k} \\
& =x^{m-1}\left(P_{2}+C_{1} x^{k+1}\right),
\end{aligned}
$$

if $k\left(k^{2}-1\right) \neq 0$.

We assume that $k \in \mathbb{C} \backslash\{-1,0,1\}$. From the recursive integration of the system (15) we deduce that the vector a has the following components

$$
\begin{aligned}
& a_{0}=x^{m} C_{0}, \\
& a_{1}=P_{m+1}+C_{1} x^{k+m}=x^{m-1} P_{k+1}, \\
& a_{2}=P_{k+m+1}+C_{2} x^{2 k+m}=x^{m-2} P_{2 k+2}, \\
& \vdots \\
& a_{S-1}=P_{(S-2) k+m+1}+C_{S-1} x^{(S-1) k+m}=x^{m-S+1} P_{(S-1) k+S-1}, \\
& a_{S}=P_{(S-1) k+m+1}+C_{S} x^{S k+m}=x^{m-S} P_{S k+S},
\end{aligned}
$$

Since $a_{0} \neq 0$, we get that $C_{0} \neq 0$. In order to simplify the proof and to avoid many cases, we assume that all the integration constants $C_{j}$ for $j=1, \ldots, S$ are non-zero, otherwise working in a similar way we should get that some of the polynomials $P_{l}$ which appear in (20) would have lower degree, and this does not perturb the general bound for the $\operatorname{deg} g$. Therefore, since the $a_{j}$ must be polynomials, $k$ is an integer and as a consequence $P_{j}=P_{j}(x)$ is a polynomial of degree $j$ in $x$. Hence we obtain the $\operatorname{deg} a_{j} \leq k j+m$, for $j=0,1,2, \ldots S$. Therefore $\operatorname{deg} g \leq k S+m=q_{0} S+\alpha-q_{0} S=\alpha$. On the other hand by considering that

$$
g=\sum x^{m-j} y^{S-j} P_{j(k+1)}=x^{m-S} \sum(x y)^{S-j} P_{j(k+1)}=0,
$$

and in view that the curve must be irreducible we obtain that $m=\alpha-k S=S$, therefore $\alpha=S(k+1)$. Clearly if $m-S<0$, then $k S+m<S(k+1)=\alpha$. So $\operatorname{deg} g \leq(k+1) S$. We are interesting in determining the biggest finite upper bound of the degree of the polynomial $g$.

We shall study the last equation of system (15). We prove that if $C_{S} \neq 0$ then the curve $g=0$ has the cofactor $K=\alpha y$. Indeed inserting $a_{S}$ and $a_{S-1}$ in the last equation of system (15) we obtain that

$$
\beta C_{S} x^{S k+m+1}+\gamma C_{S} x^{S k+m}+P_{S k+m-1}(x)=0, \quad \text { if } \quad k \geq 3 .
$$

Hence $\beta=\gamma=0$ and the cofactor is $\alpha y$.

We prove that if $C_{S} C_{S-1} \neq 0$ hence $k=3$. Indeed, from the last equation of system (15) we obtain that the polynomials $a_{S}$ and $a_{S-1}$ are such that

$$
\frac{d a_{S}}{d x}+q_{2} a_{S-1}=0 .
$$


After the integration we get

$$
a_{S}=q_{22}\left(\frac{C_{S-1}}{k(S-1)+m} x^{(S-1) k+3+m}+\ldots\right) .
$$

On the other hand the polynomial $a_{S}$ has degree $k S+m$, therefore

$$
C_{S} x^{S k+m}+\ldots=q_{22}\left(\frac{C_{S-1}}{k(S-1)+3+m} x^{(S-1) k+3+m}+r_{0} x^{k(S-2)+m+3} \ldots\right),
$$

where $r_{0}$ is a real constant. Hence if $C_{S} C_{S-1} \neq 0$, then $k=q_{0}=3$. Since $\operatorname{deg} g \leq(k+1) S=4 S$.

If $C_{S-1}=0$ and $r_{0} \neq 0$, then $k=3 / 2$, and consequently $\operatorname{deg} g \leq(3 / 2+1) S \leq$ $3 S$. Clearly if $C_{S}=0$ then, from (21), it follows that $C_{S-1}=0$, thus $\operatorname{deg} g<4 S$. In this case working as in the case that the constants $C_{j}$ were not zero with $m=0$ we should get for the curve

$$
g=y^{S}+a_{1} y^{S-1}+a_{2} y^{S-2}+\ldots a_{s}=0,
$$

that $\operatorname{deg} g \leq 3 S$.

Now we assume that $k=q_{0}=0$. The recursive integration of system (15) produces the following polynomial solutions

$$
a_{0}=x^{\alpha}, \quad a_{j}=r_{j} x^{\alpha+j}+x^{\alpha-j} P_{2 j-1}=x^{\alpha-S}\left(r_{j} x^{S+j}+x^{S-j} P_{2 j-1}\right),
$$

for $j=1,2, \ldots S$, where $r_{j}$ are rational function in the variables $q_{11}, q_{12}, q_{21}, q_{22}, q_{20}, q_{10}, \alpha, \beta$, and $P_{m}(x)$ is a polynomial of degree $m$ in the variable $x$. Note that $\operatorname{deg} a_{j} \leq$ $\alpha+j$. The polynomial $g$ becomes

$$
g=x^{\alpha-S} \sum_{j=0}^{S}\left(r_{j} x^{S+j}+x^{S-j} P_{2 j-1}\right) y^{S-j}=x^{\alpha-S} \sum_{j=0}^{S}(x y)^{S-j}\left(r_{j} x^{2 j}+P_{2 j-1}\right),
$$

where $r_{0}=1$ and $P_{-1}(x)=0$. In view that the curve $g=0$ is irreducible then $\alpha=S$. If $\alpha-S<0$, then $\operatorname{deg} g \leq \alpha+S<2 S$.

If $k=1$, then system (15) becomes

$$
\begin{aligned}
x a_{0}^{\prime} & =m a_{0}, \\
x a_{j+1}^{\prime} & =(m+j+1) a_{j+1}+ \\
& \left(\left(\beta-(S-j) q_{11}\right) x+\gamma-(S-j) q_{10}\right) a_{j}-a_{j}^{\prime}-(S-j) q_{2} a_{j-1},
\end{aligned}
$$

for $j=1,2, \ldots S$, where $a_{-1}=0$. Hence after integration it is easy to show that

$$
\begin{aligned}
& a_{0}=C_{0} x^{m}=x^{m-1} P_{1}, \\
& a_{1}=C_{1} x^{m+1}+\left(S q_{10}-\gamma\right) x^{m}+\frac{m}{2} x^{m-1}=x^{m-1} P_{2}, \\
& a_{2}=x^{m-1} P_{3}, \\
& \vdots \\
& a_{S}=x^{m-1} P_{S+1},
\end{aligned}
$$


where $P_{j}=P_{j}(x)$ is a polynomial of degree $j$ in the variable $x$. Hence

$$
g=x^{m-1} \sum_{j=0}^{S} P_{j+1}(x) y^{S-j}=0 .
$$

By considering that this curve must be irreducible, we have that $m=\alpha-S=1$. As a consequence $S=\alpha-1<\alpha$ and $\operatorname{deg} g \leq S+m=S+1$.

For the case when $k=-1$ system (15) takes the form

$$
\begin{aligned}
& x a_{0}^{\prime}=(\alpha+S) a_{0}, \\
& \left.x a_{1}^{\prime}=(\alpha+S-1) a_{1}+\left(\left(\beta-S q_{11}\right) x+\gamma-S q_{10}\right)\right) a_{0}-a_{0}^{\prime}, \\
& \left.x a_{2}^{\prime}=(\alpha+S-2) a_{2}+\left(\left(\beta-(S-1) q_{11}\right) x+\gamma-(S-1) q_{10}\right)\right) a_{1}-a_{1}^{\prime},
\end{aligned}
$$

After the recursive integrations we obtain that the polynomial solutions exist in particular if $\alpha+S=0$. In this case we obtain that $\operatorname{deg} a_{j} \leq j$, and as a consequence $\operatorname{deg} g \leq S$.

In short Theorem 3 is proved.

Remark 13. Let

$$
R(x)=p_{2} \frac{d a_{S}}{d x}-\left((\beta x+\gamma) a_{S}-q_{2} a_{S-1}\right)=\sum_{j=0}^{4 S+1} A_{j} x^{j},
$$

be a polynomial of degree at most $4 S+1$ in the variable $x$, where

$$
A_{j}=A_{j}\left(q_{0}, q_{11}, q_{10}, q_{22}, q_{21}, q_{20}, \alpha, \beta, \gamma, C_{0}, C_{1}, \ldots, C_{S}\right),
$$

for $j=0,1, \ldots, 4 S+1$. To determine the exact degree of the invariant curve $g=0$ in all the cases studied in the proof of Theorem 3, it is necessary that the polynomial $R(x)$ be zero. This holds if and only if all the coefficients are zero, i.e. $A_{j}=0$ for $j=0,1, \ldots, 4 S+1$. The compatibility of all these equations is require. Working a little it is possible to reduce the system $A_{j}=0$ to a polynomial system in the variables $q_{0}, q_{11}, q_{10}, q_{22}, q_{21}, q_{20}, \alpha, \beta, \gamma, C_{0}, C_{1}, \ldots, C_{S}$. These polynomials in general have high degree and it is not easy to work with them for proving that they do not have solution, and consequently the deg $g$ (which from the proof of Theorem 3 must be a multiple of $S$ smaller than or equal to $4 S$ ) seems that must be $\leq 3 S$.

In view of this remark and the comments later on we do the Conjecture 4 and Conjecture 5 .

These conjectures are supported mainly by the following facts. First we are able to show that for $S=1,2, \ldots, 5$ there are irreducible invariant algebraic curves $g=0$ of degree $3 S$ for convenient quadratic system (19). This curve has a cofactor $K=3 S y$. On the other hand without loss of generality we can suppose that the given invariant curve has the form (22) for which the $\operatorname{deg} g \leq 3 S$. 
Second we determine the more general quadratic systems (19) having some focus. Thus we get

$$
\begin{aligned}
& q_{0}=3, \quad q_{22}=\frac{84 a e^{2}-36 e^{2}-25 e^{4}-h^{2}}{288}, \quad q_{10}=0, \\
& q_{11}=a, \quad q_{20}=\frac{36 a^{2}-36 a e^{2}+e^{4}+h^{2}}{48 e^{2}},
\end{aligned}
$$

where $e h \neq 0$. The points $\left(\frac{\sqrt{6}}{e},-\frac{e}{\sqrt{6}}\right)$ and $\left(-\frac{\sqrt{6}}{e}, \frac{e}{\sqrt{6}}\right)$ are singular points of the corresponding quadratic system with eigenvalues $\left(6 a-7 e^{2} \pm i h\right) /(2 \sqrt{6} e)$ and $\left(-6 a+7 e^{2} \pm i h\right) /(2 \sqrt{6} e)$ respectively, so they are foci, and consequently these quadratic system do not admit a rational first integral (see for instance [12]).

We study the particular systems of (15) satisfying (23) with $S=4$, and we obtain the family of quadratic systems

$$
\dot{x}=x y+1, \quad \dot{y}=3 y^{2}-10 a x y-150 a^{2} x^{2}+59 a,
$$

where $a$ is a nonzero parameter, which admits the following family of invariant algebraic curves of degree 12

$$
\begin{aligned}
& -781250000 a^{8} x^{12}+312500000 a^{7} x^{10}-62500000 a^{6} y x^{9}-159375000 a^{6} x^{8}- \\
& 3750000 a^{5} y x^{7}+230375000 a^{5} x^{6}+375000 a^{4} y^{2} x^{6}-9975000 a^{4} y x^{5}- \\
& 82923125 a^{4} x^{4}-4215000 a^{3} y^{2} x^{4}+281000 a^{2} y^{3} x^{3}+5291500 a^{3} y x^{3}+ \\
& 3833820 a^{3} x^{2}+210750 a^{2} y^{2} x^{2}-6860 a y^{3} x-129960 a^{2} y x+343 y^{4}+ \\
& 110592 a^{2}+12348 a y^{2}=0 .
\end{aligned}
$$

The singular points of system (14) are foci, hence it has no rational first integrals. From this example we show that the degree of the invariant algebraic curve of the studied quadratic systems without rational first integral is greater than or equal to 12 .

\section{5. $C^{1}$ VECTOR FIELDS WITH ONLY ONE INVARIANT CURVE OF THE FORM$$
y=y(x)
$$

Now we determine the differential system which admits a unique invariant curve

$$
G=a_{0}(x) y+a_{1}(x)=0, \quad a_{0}(x) \neq 0 .
$$

Proposition 14. A differential system having the orbit $g=y-y_{1}(x)=0$, where $y_{1}=y_{1}(x)$ is a $\mathcal{C}^{1}$ function, can be written as

$$
\dot{x}=\lambda+\mu g=P, \quad \dot{y}=\lambda y_{1}^{\prime}+\nu g=Q,
$$

where $\lambda, \mu$ and $\nu$ are arbitrary $\mathcal{C}^{1}$ functions.

Proof. We set $\mathcal{X}=(P, Q)$. First we prove that the curve $g=0$ is invariant of the vector field $\mathcal{X}$. Indeed $\mathcal{X}(g)=\left(-\mu y_{1}^{\prime}+\nu\right) g$. Hence $g=0$ is an invariant curve of the differential system associated to the vector field $\mathcal{X}$. Let $\mathcal{Y}=$ 
$\left(Y_{1}(x, y), Y_{2}(x, y)\right)=\left(Y_{1}, Y_{2}\right)$ be another vector field with the given invariant curve, i.e.

$$
\left.\mathcal{Y}(g)\right|_{g=0}=Y_{2}\left(x, y_{1}(x)\right)-Y_{1}\left(x, y_{1}(x)\right) y_{1}^{\prime}(x)=0 .
$$

Taking

$$
\begin{aligned}
& \lambda(x, y)=Y_{1}(x, y)-g(x, y) \mu(x, y), \\
& \nu(x, y)=\frac{Y_{2}(x, y)-y_{1}^{\prime}(x) Y_{1}(x, y)}{g(x, y)}+y_{1}^{\prime}(x) \mu(x, y),
\end{aligned}
$$

and inserting them into $(26)$ we obtain the vector field $\mathcal{Y}$.

\section{VERIFICAR EL SIGUIENTE REMARK}

Remark 15. We suppose that in (26)

$$
\lambda=a_{0} \tilde{\lambda}, \quad \mu=a_{0} \tilde{\mu}, \quad \nu=a_{0} \tilde{\nu}+\frac{a_{0}^{\prime}}{a_{0}} \tilde{\lambda} .
$$

Then system (26) takes the the form

$$
\dot{x}=\tilde{\lambda} a_{0}+\tilde{\mu} g=P, \quad \dot{y}=-\tilde{\lambda}\left(a_{0}^{\prime} y+a_{1}^{\prime}\right)+\tilde{\nu} g=Q,
$$

which is the most general system for which the curve $G=0$ is invariant.

\section{QuAdRATIC SYSTEM WITH A UNIQUE INVARIANT ALGEBRAIC CURVE WITH ONE BRANCH}

Now we consider the vector field $\mathcal{X}$ associated to the quadratic system

$$
\dot{x}=p_{2}, \quad \dot{y}=q_{0} y^{2}+q_{1} y+q_{2},
$$

where $p_{2} \neq 0$ and $q_{j}$ are polynomials of degree $j$ in the variable $x$. We assume that $\mathcal{X}$ has the curve $G=0$, given in (25) with $a_{0}$ and $a_{1}$ polynomials, as an invariant algebraic curve. System (15) is valid in this case.

Proposition 16. The algebraic curve $G=a_{0}(x) y+a_{1}(x)=0$ is invariant for the quadratic system (27) with cofactor $K=\alpha y+\beta x+\gamma$ if and only if $\alpha a_{1}=a_{1}(x)=p_{2} a_{0}^{\prime}-r a_{0}$, where $r=\left(\beta-q_{11}\right) x+\gamma-q_{10}$ and $a_{0}=a_{0}(x)$ is a polynomial solution of the Fucshian equation

$$
w^{\prime \prime}+\varrho_{1} w^{\prime}+\varrho_{2} w=0,
$$

where

$$
\varrho_{1}=\frac{p_{2}^{\prime}-(\beta x+\gamma)-r}{p_{2}}, \quad \varrho_{2}=\frac{(\beta x+\gamma) r+q_{2}-r^{\prime} p_{2}}{p_{2}^{2}} .
$$

Proof. Under the given conditions system (15) takes the form

$$
\alpha-q_{0}=0, \quad p_{2} a_{0}^{\prime}=\alpha a_{1}+r a_{0}, \quad p_{2} a_{1}^{\prime}=(\beta x+\gamma) a_{1}-q_{2} a_{0} .
$$

After differentiation of the previous second equation, and using the third equation we get

$$
p_{2}^{2} a_{0}^{\prime \prime}+\left(p_{2} p_{2}^{\prime}-(\beta x+\gamma) p_{2}-r p_{2}\right) a_{0}^{\prime}+\left((\beta x+\gamma) r+q_{2}-r^{\prime} p_{2}\right) a_{0}=0 .
$$


We observe that the above Fucshian equation admits a polynomial solution if $q_{2}=\left(\kappa+r^{\prime}\right) p_{2}-(\beta x+\gamma) r$. Indeed in this case the obtained equation takes the form

$$
p_{2} a_{0}^{\prime \prime}+\left(p_{2}^{\prime}-(\beta x+\gamma)-r\right) a_{0}^{\prime}+\kappa a_{0}=0 .
$$

Hence $a_{0}$ is an orthogonal polynomial because the degrees of $p_{2}=p_{2}(x), p_{2}^{\prime}-$ $(\beta x+\gamma)-r$ and $\kappa$ are 2,1,0 respectively (see for instance [1])

For a precise definition of a family of orthogonal polynomials see [1]. A very important class of orthogonal polynomials $f_{0}, f_{1}, \ldots f_{n}, \ldots$ are the ones satisfying the differential equation

$$
p(x) f^{\prime \prime}+q(x) f^{\prime}+r f=0,
$$

where $p=p(x)$ is a polynomial of degree at most two, $q=q(x)$ is a linear polynomial, and $r$ is nonzero constant.

Proposition 17. Let $f$ be an orthogonal polynomial satisfying equation (28).

(i) There exists a quadratic polynomial differential system having the invariant algebraic curve $\tilde{G}=f^{\prime}(x) y+f(x)=0$.

(i) There exists a polynomial differential system of degree 2,3 or 4 having the invariant algebraic curve $G=f(x) y+f^{\prime}(x)$.

Proof. ¿From (7) the vector field with the invariant algebraic curve $\tilde{G}=0$ can be written as

$$
\begin{aligned}
& \dot{x}=\lambda(x, y) f^{\prime}(x)+\mu(x, y)\left(f^{\prime}(x) y+f(x)\right), \\
& \dot{y}=-\lambda(x, y)\left(f^{\prime \prime}(x) y+f^{\prime}(x)\right)+\nu(x, y)\left(f^{\prime}(x) y+f(x)\right),
\end{aligned}
$$

where $\lambda, \mu$ and $\nu$ are polynomials. Taking

$$
\lambda=-y \mu, \quad \nu=\mu\left(\frac{q y}{p}-1\right), \quad \mu f(x)=p(x),
$$

from (28) we obtain that the differential system takes the form

$$
\dot{x}=p(x), \quad \dot{y}=-r y^{2}+q(x) y-p(x) .
$$

The cofactor of the curve $\tilde{G}=0$ is $-r y$. After the change $-r y \succ y$ we obtain the differential system

$$
\dot{x}=p(x), \quad \dot{y}=y^{2}+q y+p r .
$$

The cofactor in this case is $y$. This system admits three, two or one invariant algebraic curve depending of degree of $p(x)$. Hence statement (a) is proved.

In particular the quadratic differential system

$$
\dot{x}=1, \quad \dot{y}=2 n+2 x y+y^{2},
$$

for any $n \in \mathbb{N}$ admits a unique irreducible nonsingular in the affine plane invariant algebraic curve $g=y H_{n}(x)+2 H_{n}^{\prime}(x)$, where $H_{n}$ is the physicists' Hermite polynomial (for more details see [3]). 
From (7) the differential system

$$
\begin{aligned}
& \dot{x}=\lambda(x, y) f(x)+\mu(x, y)\left(f(x) y+f^{\prime}(x)\right), \\
& \dot{y}=-\lambda(x, y)\left(f^{\prime}(x) y+f^{\prime \prime}(x)\right)+\nu(x, y)\left(f(x) y+f^{\prime}(x)\right),
\end{aligned}
$$

has the invariant curve $G=f(x) y+f^{\prime}(x)$, where again $\lambda, \mu$ and $\nu$ are arbitrary polynomials. Hence by choosing

$$
\lambda=-y \mu, \quad f^{\prime} \mu=p, \quad p \nu-r \mu=0,
$$

and in view of (28) we finally deduce the system

$$
\dot{x}=p(x), \quad \dot{y}=p(x) y^{2}-q(x) y+r .
$$

This system has degree two, three or four and admits one, two or three invariant algebraic curves depending on the degree of polynomial $p(x)$. Moreover the cofactor of $G=0$ is $p(x) y-q(x)$.

From Proposition 17 it follows that there exist polynomial differential systems with invariant algebraic curves of arbitrary high degree.

\section{ON The 16Th Hilbert PROBlem fOR Limit CYClES ON SingulaR INVARIANT ALGEBRAIC CURVE}

One of the motivations of this paper was to study the 16th Hilbert problem for limit cycles on singular invariant algebraic curves. As it follows from the results exposed in [10] and [11] for solving this problem it is necessary to determine the maximum degree of the invariant algebraic curves (Poincaré's problem). It is well known that if the invariant algebraic curve $g=0$ of a polynomial vector field of degree $n$ is non-singular in $\mathbb{C} P^{2}$ then $\operatorname{deg} g \leq n+1$ (see for instance [4]). Additionally in that paper the authors gave the following result: if all the singularities on the invariant algebraic curve $g=0$ are double and ordinary, then $\operatorname{deg} g \leq 2 n$.

If the algebraic curve is of nodal type, i.e. it is singular and all its singularities are normal crossing type (that is at any singularity of the curve there are exactly two branches of $g=0$ which intersect transversally), then $\operatorname{deg} g \leq n+2$ (see for more deatails [2]).

To determine an upper bound for the degree of a singular invariant algebraic curve is in general an open problem. In this direction there are the following two results.

Theorem 18 ([16]). If there exists an integer $\kappa$ such that for all polynomial vector fields of degree $n$ having the singular invariant algebraic curve $g=0$, we have that $\left(g, \partial_{y} g\right) \leq \kappa$, where $\left(g, \partial_{y} g\right)$ is the intersection number of the curves $g=0$ and $\partial_{y} g=0$ at a point of $g \cap \partial_{y} g$, then

$$
\operatorname{deg} g \leq \frac{4+2 n+\kappa+\sqrt{(4+2 n+\kappa)^{2}+16 \kappa n^{2}}}{4} .
$$




\section{EL PÁRRAFO SIGUIENTE NECESITA MÁS DETALLES}

By considering that $\forall \kappa \geq 1$

$$
\frac{4+2 n+\kappa+\sqrt{(4+2 n+\kappa)^{2}+16 \kappa n^{2}}}{4} \leq(\kappa+1)(n+1)
$$

we obtain the following upper bound for the degree of invariant algebraic curve

$$
\operatorname{deg} g \leq(\kappa+1)(n+1), \quad \kappa \geq 1 .
$$

Theorem $19([8])$. Let $\mathcal{X}=P \partial_{x}+Q \partial_{y}$ be a polynomial vector field of degree $n$ over $\mathbb{K}[x, y]$ and suppose that in some extension $\mathbb{K}^{\prime}$ of $\mathbb{K}$ the vector field has an invariant algebraic curve $g=0$ where $g \in \mathbb{K}^{\prime}[x, y]$ is a square-free polynomial. Let $K$ be the maximum of the Tjiurina numbers of the singularities that the curve has in the projective plane. Then

$$
\operatorname{deg} g \leq \frac{1+\sqrt{1+4 K})(n+2)}{2} .
$$

These last two theorems and the results stated in this paper show that in general for a singular invariant algebraic curve its degree can be arbitrary high (see Remark 11).

\section{EXPLICAR MEJOR EL PÁRRAFO SIGUIENTE}

Clearly if $K=\kappa(\kappa+1)$ then

$$
\operatorname{deg} g \leq(1+\kappa)(n+1) \leq \frac{1+\sqrt{1+4 K})(n+2)}{2}=(1+\kappa)(n+2) .
$$

In particular for the quadratic vector fields we obtain the following upper bound for the degree of invariant algebraic curve with $S$ branches (see Theorem 3)

$$
\operatorname{deg} g \leq 3 S, \quad \kappa=1-S
$$

and

$$
\operatorname{deg} g \leq 4 S, \quad K=S(S-1) .
$$

The solution of 16th Hilbert problem for limit cycles on generic, nonsingular and nodal algebraic curves are given respectively in [10], [11][17].

\section{ACKNOWLEDGEMENTS}

The first author is partially supported by a MCYT/FEDER grant number MTM2009-03437, by a CICYT grant number 2009SGR-410 and ICREA Academia. The second author was partly supported by the Spanish Ministry of Education through projects DPI2007-66556-C03-03, TSI2007-65406-C03-01 "E-AEGIS" and Consolider CSD2007-00004 "ARES". 


\section{REFERENCES}

[1] M. Abramowitz And I. Stegun, Handbook of Mathematical Functions with Formulas, Graphs, and Mathematical Tables, New York, Dover, 1965.

[2] D. Cerveau And A. Lins Neto, Holomorphic foliations in $\mathbb{C} \times \mathbb{P}^{2}$ having an invariant algebraic curve, Ann. of Math.140 (1994), 289-294.

[3] J. Chavarriga AND M. Grau, A family of non-Darboux integrablequadratic polynomial differential systems with algebraic solutions of arbitrary high degree, Applied Mathematics Letters 16 (2003), 833-837.

[4] J. ChavarRiga AND J. LliBRe, Invariant algebraic curves and rational first integrals for planar polynomial vector fields, J. Differential Equations 169 (2001), 1-16.

[5] C. Christopher, J. Llibre, C. Pantazi And X. Zhang, Darboux integrability and invariant algebraic curves for planar polynomial systems, J. of Physics A: Math. and Gen. 35 (2002), 2457-2476.

[6] W. Fulton, Algebraic curves, New York, W.A. Benjamin Inc., 1969.

[7] A.Gasull, Sheng Li And J. Llibre, Chordal quadratic systems, Rocky Mountain J. of Math. 16 (1986), 751-782.

[8] M'Hammed El Jahoui, On the plane polynomial vector fields and the Poincaré problem, Electronic J. Differential Equations 2002, 1-23.

[9] J. LliBRE, Integrability of polynomial differential systems, Handbook of Differential Equations, Ordinary Differential Equations, Eds. A. Cañada, P. Drabek and A. Fonda, Elsevier, 2004, pp. 437-533.

[10] J. Llibre, R. Ramírez And N. Sadovskaia, On the 16th Hilbert problem for algebraic limit cycles, J. Differential Equations 248 (2010), 1401-1409.

[11] J. Llibre, R. Ramírez And N. Sadovskaia, On the 16th Hilbert problem for limit cycles on nonsingular algebric curves, J. Differential Equations, to appear.

[12] W. Li, J. LliBre, X. ZHANG, Planar vector fields with generalized rational first integrals, Bull. Sci. Math. 125 (2001), 341-361.

[13] R. RAmíREz AND N. SAdovskaia, Inverse approach in the study of ordinary differential equations, preprint Universitat Rovira i Virgili (2008), 1-49.

[14] R. RAmirez AND N. SAdovskaia, Differential equations on the plane with given solutions, Collect. Math. 47 (1996), 145-177.

[15] N. Sadovskaia, Problemas inversos en la teoría de las ecuaciones diferenciales ordinarias, Ph.D., Universitat Politécnica de Catalunya, Barcelona 2002 (in Spanish).

[16] A. Tsygventsev, Algebraic invariant curves of plane polynomial differential systems, J. Phys. A: Math. Gen. 34 (2001), 663-672.

[17] X. ZHANG, The 16th Hilbert problem on algebraic limit cycles, (2010), preprint.

[18] G. Wilson, Hilbert's sixteenth problem, Topology 17 (1978), 53-74.

${ }^{1}$ Departament de Matemàtiques, Universitat Autònoma de Barcelona, 08193

Bellaterra, Barcelona, Catalonia, Spain.

E-mail address: jllibre@mat.uab.cat

2 Departament D'Enginyeria Informàtica i Matemàtiques, Universitat Rovira i Virgili, Avinguda dels Països Catalans 26, 43007 Tarragona, Catalonia, SPAIN.

E-mail address: rafaelorlando.ramirez@urv.cat

3 Departament de Matemàtica Aplicada II, Universitat Politècnica de Catalunya, C. Pau Gargallo 5, 08028 Barcelona, Catalonia, Spain.

E-mail address: natalia.sadovskaia@upc.edu 